\title{
SEQUENTIAL SAMPLING OF INSECT POPULATIONS
}

\author{
By W. G. H. IVES 2
}

M.W. W. G. H. Ives obtained his B.S.A. degree at the University of Maniloba in 1951 majoring in Entomology. He then proceeded to a Masters of Science Degree in Statistics at lowa State College, Ames, Iowa in 1953.

He bas been employed by the Department of Agriculture, Division of Forest Biology since May, 1951, and dutring the stmmers of 1949 and 50 was witb tbis Department as a student assistant.

\section{INTRODUCTION}

The fundamental difference between conventional and sequential sampling is that in conventional sampling a decision is based on the data contained in the sample as a whole, while in sequential sampling a decision is made after each unit is drawn. Consequently, the sample size in sequential sampling varies with each sample drawn, while in conventional sampling the sample size may be regarded as fixed, since it must be chosen in advance. Although the sample size varies in sequential sampling, the average size of sample required to attain an equal degree of accuracy is usually considerably smaller than in conventional sampling. The saving in number of observations obtained by the use of sequential sampling may be as great as 50 per cent.

\section{SOME Fundamental Concepts}

A brief outline of the principles underlying conventional and sequential sampling is needed to explain why sequential sampling may require a smaller number of observations to reach a decision. In both types of sampling there are three variables involved.

These are:

a-the sample size;

a the probability of rejecting $\mathrm{H}_{0}$ (the null hypothesis) when $\mathrm{H}_{0}$ is true ('Type I Error);

$\beta$-the probability that $\mathrm{H}_{0}$ will be accepted when $\mathrm{H}_{\mathbf{1}}$ (the alternative hypothesis) is true (Type II Error).

The power of the test is equal to $1-\beta$.

In conventional sampling, the values of $\mathrm{n}$ and $\alpha$ are chosen in advance, and the test that gives the best protection against making a Type II Error is used. This protection is obtained by using the test that gives the best power for the hypothesis being tested, which gives the smallest $\beta$. A decision is made on the basis of the sample data as a whole, and the null hypothesis is accepted or tejected at the $\alpha$ confidence level.

In sequential sampling $\alpha$ and $\beta$ are chosen in advance and $x$ is allowed to vary. The sample is drawn one unit at a time, and at each draw a decision is made. The null hypothesis may be accepted, rejected, or no decision reached. If no decision is reached, the sampling is continued. If either of the other events occurs, sampling is terminated and a decision is reached at the $\alpha$ and $\beta$ confidence levels.

\footnotetext{
1 Contribution No. 168, Forest Biology Division, Science Service, Department of Agricultare,

2 Agricultural Research Officer, Forest Biology Laboratory, Winnipeg, Manitoba
} 


\section{EXAMPLES OF SEQUENTIAL SAMPLING}

Before a sequential sampling scheme can be applied to estimate the density of a given insect population, something must be known about its distribution. Two distributions commonly encountered in biological work are the Binomial and the Normal. The Binomial distribution applies to a series of individual observations from a discrete population, in which each observation may be placed in one of two mutually exclusive classes. The Normal distribution is a continuous distribution, but many discrete distributions approach normality as the sample size increases.

\section{BinOMial Distribution}

An example of sequential sampling from a Binomial distribution is the estimation of the ratio of larch sawfly oviposition sites to total number of new shoots. As larch sawfly adults lay their eggs in new shoots of tamarack; this characteristic may be used to estimate the insect's abundance. The distribution of oviposition sites in relation to total shoots appears to be random. When a series of individual observations is made, the ratio should follow the Binomial distribution. Before sampling is begun, the decisions to be made must be stated. Suppose it is decided to classify the ratio into one of the categories of light, medium, or heavy, in terms of probable defoliation. To set up these categories, sufficient information from previous sampling must be available to decide what values of the ratio (hereafter referred to as $\mathrm{p}$ ) are to be grouped into each category. For any given value of $p$, the resulting defoliation of vigorous trees will probably be higher than of less vigorous trees, due to differences in the ratio of new shoots to amount of foliage. Vigorous trees are believed to have a higher ratio of new shoots to amount of foliage than less vigorous trees. No data in support of this hypothesis are available at present, but observations made in the field indicate this trend. Further information on this phase of the problem is required, if the use of the ratio of oviposition sites to total shoots is to have much meaning in terms of actual defoliation.

Assuming that suitable values have been chosen for $p$ and for the confidence levels $\alpha$ and $\beta$, a chart is prepared like that shown in Figure 1. The values of $\mathrm{p}$ used in calculating the formulae for the lines in Figure $I$ were grouped as follows:

(1) light-less than .10;

(2) medium-greater than .15 but less than .25;

(3) heavy-greater than .30 .

These values are largely hypothetical at the present state of knowledge. Ik should be noted that there are gaps between the above classifications. The wider these gaps the smaller the amount of sampling required, and vice versin, for any given set of confidence levels. The probabilities for all four errors involved were set equal to .10 . It is not intended to present the chart as a means for estimating the density of an infestation, but rather as an illustration of the method.

The actual sampling procedure is simple. A shoot is examined and classified as bearing egg scars or not, and a point is plotted on the chart. 


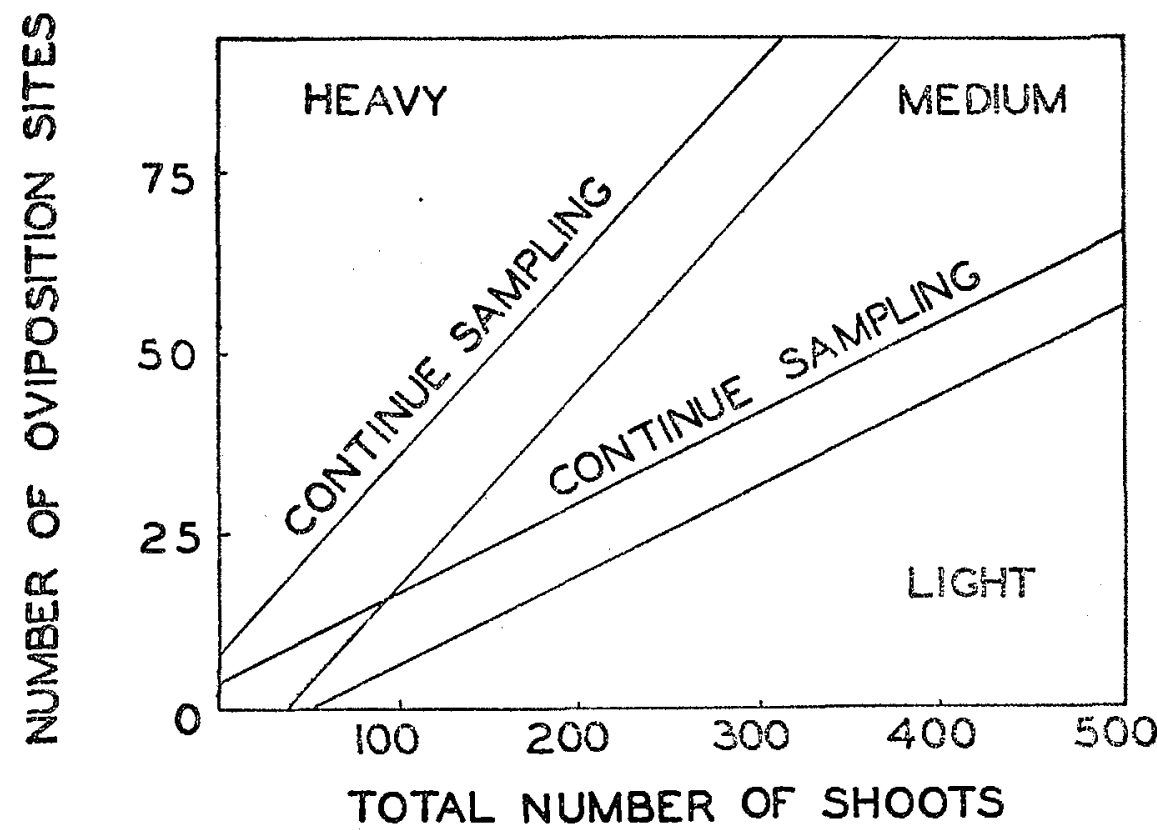

Another shoot is examined and another point is plotted, until one of the points falls into a critical region (the portions of the chart shown as light, medium and heavy). It is often more convenient to examine a group of shoots, say ten at a time, and then plot the results. This does not alter the character of the test, is generally less time consuming, and hence this procedure would usually be followed.

It is essential to avoid bias in selecting the branches and the order in which the shoots on a branch are examined. These biases may be avoided by the use of standard randomization techniques at both stages of sampling.

Previous investigation indicates that for survey purposes a random sample of two branches from the mid-crown should be selected from each of a series of trees until a decision is reached.

Normal Distribution

An example of sequential sampling from a normally distributed population was given by Stark (1952). Recently, Stark (1954) revised his population classes, but his original classes are used below to demonstrate the procedure. He presented a method of estimating population densities of the lodgepole needle miner. The number of mined needles per five-year tip was used as the index of population density. The Chi-square test was applied to the data and indicated normality. The degrees of infestation, based on the number of mined needles per tip, were grouped as follows:

(1) Light-less than 5;

(2) medium-more than 15 but less than 25 ;

(3) heavy-more than 35. 


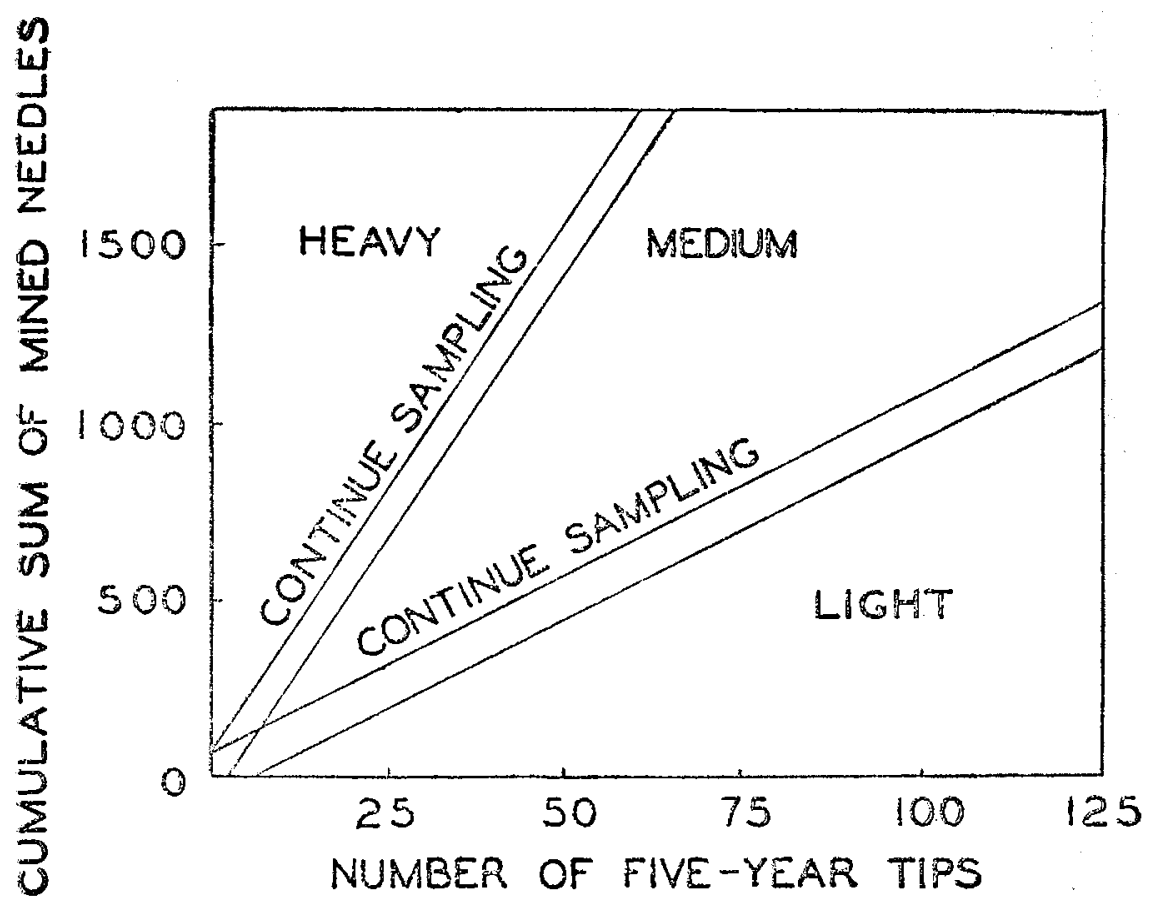

The probabilities of making the different types of errors are given by Stark and will not be discussed here. The appearance of the chart (Figure 2) is similar to that for the Binomial distribution, though different formulae were used in drawing the lines.

The sampling procedure is similar to that for the Binomial distribution, except that the cumulative sum of mined needles is plotted against the number of samples, instead of the number of oviposition sites against the total number of observations. Sampling is again terminated as soon as a point falls in one of the three critical regions.

Other Distributions

In addition to the Binomial and Normal distributions already discussed, sequential sampling schemes have been devised for Poisson, Negative Binomial and Negative Hypergeometric distributions (Oakland, 1951). However, for purposes of illustration, the two distributions already discussed should suffice.

\section{Discussion}

The chief advantage of a sequential sampling technique for estimating the density of insect populations is that a smaller sample size is usually required, for a given degree of accuracy, than with the conventional sampling method. Insect populations are usually very variable. Consequently, a large sample is required to give reasonably accurate estimates with conventional 
sampling methods. By using sequential sampling, the sample size can frequently be reduced by an appreciable amount without sacrificing accuracy. One disadvantage of the sequential sampling method is that point estimates of the density of insect populations cannot be obtained, since population levels are usually expressed in broad categories, i.e. light, medium or heavy. The number of categories that are practical in sequential sampling is limited to three or four, hence conventional sampling is required for point estimates.

\section{REFERENCES}

OAKLAND, G. B. 1951. Sequential sampling. Biometrics Unit, Science Ser, Can, Dept. of Agric. STARK, R. W. 1952. Sequential sampling of the lodgepole needle miner. For. Crron. 28:57-60.
STARK, R. W, 1954. Sequential sampling of the lodgepole needle miner. In For. Biol. Div. Bi-monthly Progress Rept. 10(2):2-8. 